\title{
Study on Large-signal Linearity and Efficiency of AlGaN/GaN MODFETs
}

\author{
Shawn S.H. Hsu, Pouya Valizadeh, Dimitris Pavlidis, Jeong S. Moon", M. Micovic ${ }^{*}$, Danny \\ Wong $^{*}$ and T. Hussain ${ }^{*}$
}

Department of Electrical Engineering and Computer Science, The University of Michigan, Ann Arbor, MI 48109-2122, USA, pavlidis@umich.edu *HRL Laboratories, 3011 Malibu Canyon Road, Malibu, CA 90265, USA

\begin{abstract}
Linearity and efficiency of AlGaN/GaN MODFETs were investigated under large-signal conditions. The poweradded-efficiency $(P A E)$ of $0.25 \times 200 \mu \mathrm{m}^{2}$ devices was found to be $\sim 33 \%$ and $\sim 45 \%$ without and with harmonic tuning. Third-order Intermodulation (IMD3) was also measured using the two-tone technique. Third-order Intercept Point (IP3) of $\sim 29 \mathrm{dBm}$ at $P_{i n}=15 \mathrm{dBm}$ was obtained at $f_{0}=5 \mathrm{GHz}(\Delta \mathrm{f}=10 \mathrm{MHz})$. Load-pull contours showed that the impedance values for best PAE and $P_{\text {out }}$ are very close. IP3 was found to be insensitive to the gate bias voltage. In addition, high efficiency and high linearity can be achieved simultaneously with the assistance of harmonic termination.
\end{abstract}

\section{INTRODUCTION}

Linearity and efficiency are important device parameters for high-frequency power amplifications. For wireless communication applications, devices with high linearity can minimize signal distortion, and high efficiency can reduce power consumption and extend battery life. GaN-based devices and circuits show impressive output power characteristics and are promising for high-frequency power amplifications. For example, Shen et al. reported an output power density of $8.4 \mathrm{~W} / \mathrm{mm}$ for GaN-based MODFETs on SiC substrate at $8 \mathrm{GHz}$ [1]. Green et al. reported a GaN-based MMIC with an output power of $6 \mathrm{~W}$ and an associated PAE of $31 \%$ at $8 \mathrm{GHz}$ [2]. Although power characteristics of AlGaN/GaN MODFETs have been reported, little has been investigated on issues related to linearity and efficiency. In this work, a systematic study on power characteristics of AlGaN/GaN MODFETs was conducted. Load-pull measurements with harmonic impedance tuning and two-tone tests were performed under different bias conditions. The results indicate that AlGaN/GaN MODFETs show significant improvement in Power-Added-Efficiency (PAE) with the assistance of harmonic tuning. In addition, the devices showed good Third-order Intermodulation (IMD3) and relatively insensitive Third-order Intercept Point (IP3) values to gate bias voltage.

\section{DEVICE CHARACTERISTICS}

The AlGaN/GaN MODFETs studied in this work, were grown on $4 \mathrm{H}-\mathrm{SiC}$ substrates using RF-assisted MBE. The measured devices had two fingers, each with a gate length of $0.25 \mu \mathrm{m}$, and a gate width of $0.1 \mathrm{~mm}$. Fig. 1 shows typical device $I_{D S}-V_{D S}$ characteristics. As can be seen, short-channel effects were not pronounced, and no obvious thermal effects were present in the measured bias range. A peak transconductance of $\sim 185 \mathrm{mS} / \mathrm{mm}$ was obtained at $V_{D S}=12 \mathrm{~V}, V_{G S}=-3 \mathrm{~V}$. In addition, the device showed maximum oscillation frequency $\left(f_{\text {max }}\right)$ of $~$ $110 \mathrm{GHz}$, and current gain cut-off frequency $\left(f_{T}\right)$ of $\sim 60$ $\mathrm{GHz}$, respectively.

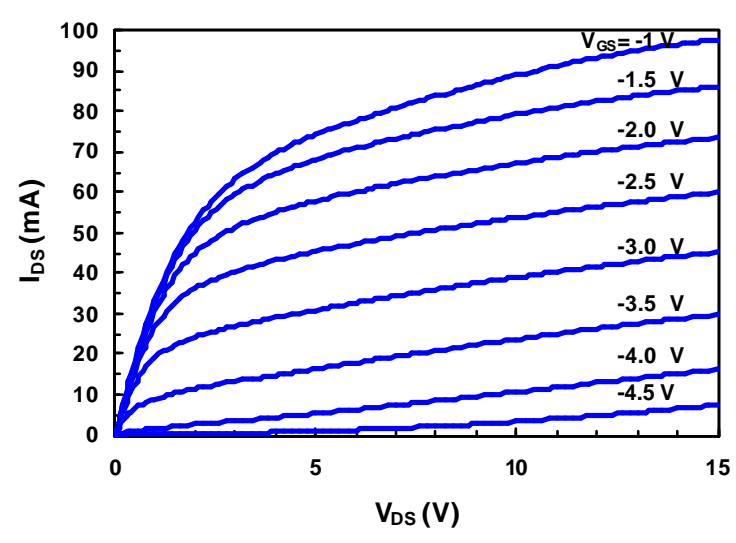

Figure 1: DC I-V characteristics of $0.25 \times 200 \mu \mathrm{m}^{2} \mathrm{AlGaN} / \mathrm{GaN}$ MODFETS.

\section{EXPERIMENTAL SETUP AND RESULTS}

Load-pull power characterization was performed at 5 $\mathrm{GHz}$ using electromechanical tuners from Focus Microwaves Inc. Both source and load tuners were employed at the fundamental frequency, and load 
harmonic tuners were used at the 2 nd and the 3rd harmonic frequencies. The harmonic tuners used were optimized at $2 f_{0}=10 \mathrm{GHz}$, and $3 f_{0}=15 \mathrm{GHz}$, and the insertion loss of the harmonic tuner was $\sim 0.2 \mathrm{~dB}$ at $f_{0}$, while it could reach $\sim 38 \mathrm{~dB}$ and $\sim-20 \mathrm{~dB}$ at $2 f_{0}$ and $3 f_{0}$, respectively. Two-tone measurements were performed using the same setup with an additional power combiner at the input. To complete the large-signal linearity study of AlGaA/GaN MODFETs, IMD3 was measured with two tones separated by $500 \mathrm{KHz}$ and $10 \mathrm{MHz}$. A spectrum analyzer was used to differentiate and measure the harmonic power spectrum from IMD3. $\mathrm{P}_{\text {out }}$, was evaluated with and without the harmonic tuners to investigate the impact of harmonic termination on PAE and IMD3.

Fig. 2 shows the power characteristics of the device when biased under $V_{G S}=-3 \mathrm{~V}$, and $V_{D S}=12 \mathrm{~V}$. The source, load and harmonic impedances were optimized for the best PAE performance $\left(\Gamma s=0.710 \angle 29.4^{\circ}, \Gamma_{L}=0.695 \angle\right.$ $19.5^{\circ}, \Gamma_{L}^{2 \text { nd }}=0.833 \angle-13.7^{\circ}$, and $\left.\Gamma_{L}^{3 \mathrm{rd}}=0.655 \angle-73.8^{\circ}\right)$. The device shows a maximum output power of 22.4 $\mathrm{dBm}$, a gain of $20.1 \mathrm{~dB}$, and PAE of $44.2 \%$, respectively. Similar gain and output power level were obtained with and without the harmonic tuners. However, an improvement of $\sim 12 \%$ on PAE with harmonic frequency terminations was found at high input power levels. Harmonic tuning allows improvement of device efficiency by changing the load line of devices [3]. Since the load line was found to be less steep when the output power is terminated properly at harmonic frequencies, the PAE increases correspondingly. The measured DC bias current levels under large-signal output conditions were 24.5 and $35.4 \mathrm{~mA}$ with and without harmonic tuner. The termination of harmonic frequencies reduces the effective DC biasing current and therefore improves PAE.

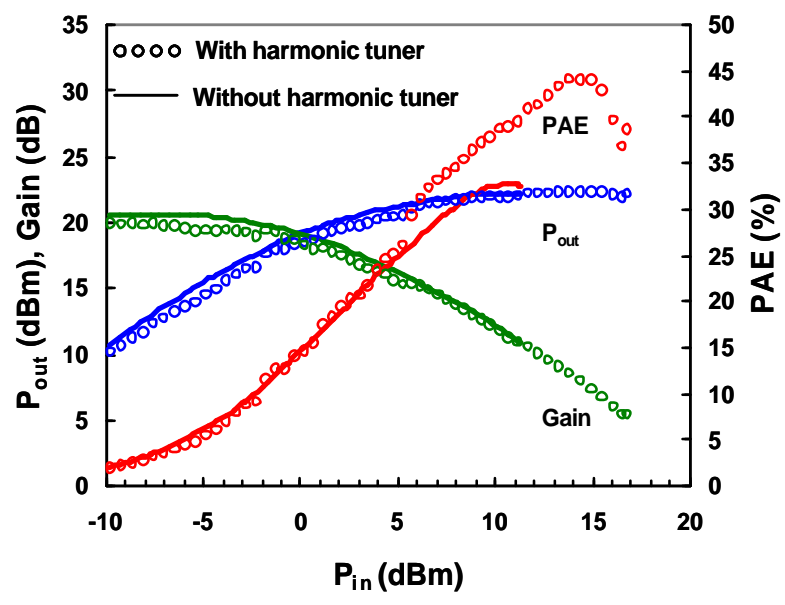

Figure 2: Power characteristics of AlGaN/GaN MODFETs $\left(0.25 \times 200 \mu \mathrm{m}^{2}\right)$ with and without output harmonic termination.
Fig. 3 shows $\mathrm{P}_{\text {out }}$ and PAE contours from load-pull measurements at an input power level of $13.7 \mathrm{dBm}$. The device was biased at $V_{G S}=-3 \mathrm{~V}$, and $V_{D S}=12 \mathrm{~V}$. Harmonic tuners were used in this case. Maximum $\mathrm{P}_{\text {out }} \sim 21.2 \mathrm{dBm}$ and PAE $\sim 35 \%$ were obtained at this input power level using load impedances of $0.685 \angle 19.1^{\circ}$ and $0.796 \angle 8.2^{\circ}$, respectively. As can be seen, the impedances of optimized $\mathrm{P}_{\text {out }}$ and PAE are close. The results indicate that the devices present high RF gain and efficiency simultaneously in the presence of similar impedances, which is a very promising characteristic for power amplifier applications.

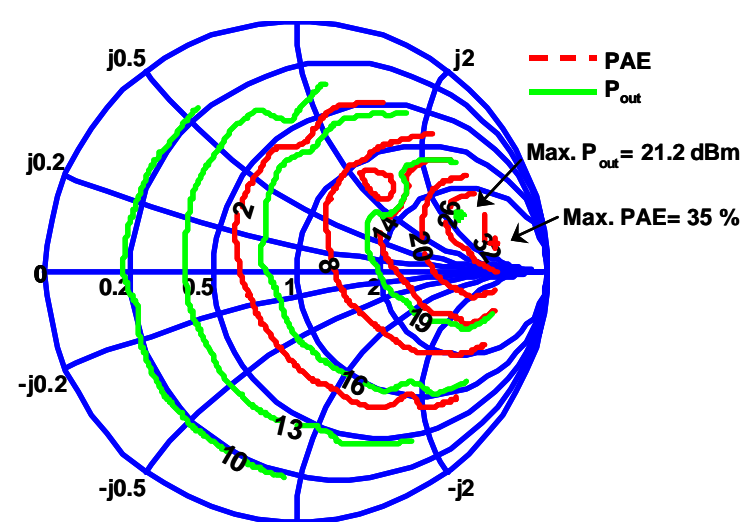

Figure 3: Load-pull measurements of PAE and Pout at $5 \mathrm{GHz}$ under $P_{\text {in }}$ of $13.7 \mathrm{dBm}$.

Fig. 4 shows the fundamental $\mathrm{P}_{\text {out }}$ and IMD3 of the devices under a two-tone test at $f_{0}=5 \mathrm{GHz}$ with $\Delta f=10$ MHz. IP3 was found by extrapolation to be $\sim 29 \mathrm{dBm}$ at an input power level of $\sim 15 \mathrm{dBm}$. Fig. 5 shows the results obtained for $\Delta f=500 \mathrm{KHz}$, and IP3 was found to be $\sim 25 \mathrm{dBm}$ at an input power of $\sim 12 \mathrm{dBm}$. The results indicate that AlGaN/GaN MODFETs show good IMD3 and IP3 values under a wide range of second tone separation. IMD3 was also measured without harmonic terminations and similar values of IMD3 and IP3 were observed. Trade offs exist in principle between linearity and efficiency. To achieve a higher PAE, one needs to operate the devices under class $\mathrm{B}$ condition which leads in lower linearity. The device enters in such cases the cut-off region during half of the operating cycle, the average DC power reduces, and the efficiency increases consequently. When the device operates under class A condition, the signal can maintain a full swing and higher linearity can be obtained, while the use of higher bias current under class A operation, results in lower efficiency. However, the results obtained with harmonic tuning in this study suggest that the termination of harmonic frequencies improves the efficiency of the devices significantly without degradation of the device linearity. Therefore, harmonic tuning can be employed 
for high efficiency and high linearity microwave power applications using the AlGaN/GaN MODFETs of this study.

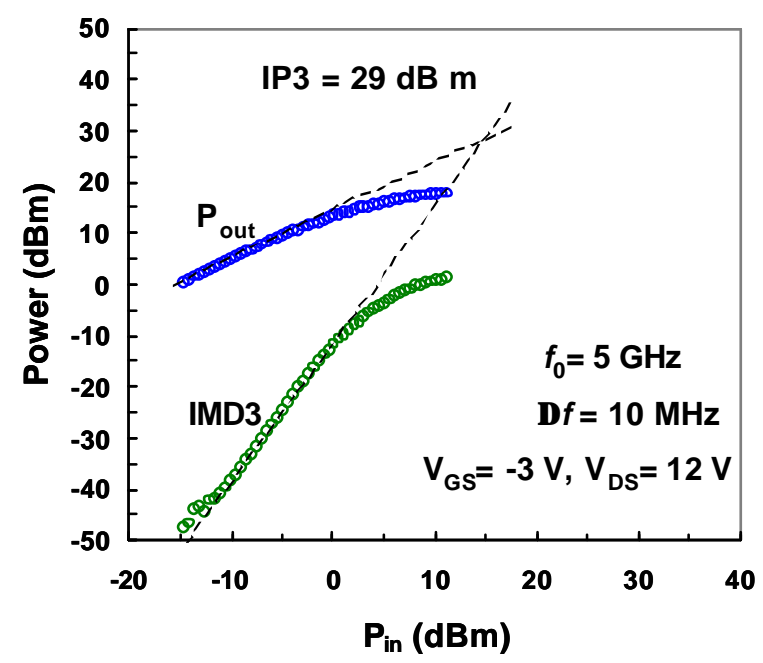

Figure 4: $P_{\text {out }}$ and IMD3 as a function of $P_{\text {in }}$ at $5 \mathrm{GHz}$ and $\Delta f=$ $10 \mathrm{MHz}$. IP3 was extrapolated to be $29 \mathrm{dBm}$.

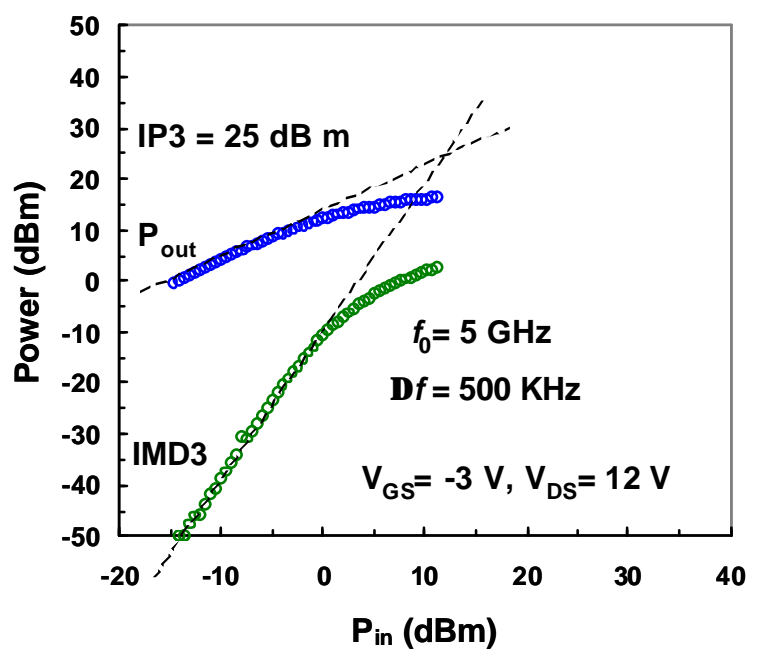

Figure 5: $P_{\text {out }}$ and IMD3 as a function of $P_{\text {in }}$ at $5 \mathrm{GHz}$ and $\Delta f=$ $500 \mathrm{KHz}$. IP3 was extrapolated to be $25 \mathrm{dBm}$.

Fig. 6 shows the IMD3 $(\mathrm{dBc})$ relative to the fundamental output power level with $\Delta f=500 \mathrm{KHz}$ under different gate bias voltages. The device was biased from Class A to Class B by changing the gate voltage from -3 $\mathrm{V}$ to $-4.5 \mathrm{~V}$. As can be seen, the IMD3 of these devices is insensitive to the gate voltage when $P_{1 n}$ is relatively small. When the gate voltage moves toward pinch-off,
IMD3 becomes smaller at high power levels. At $V_{G S}=-$ $4.5 \mathrm{~V}$, a clear notch was observed in the IMD3 versus $P_{\text {in }}$ characteristics. This can be explained by the cancellation effect from the nonlinearities of transconductance and output conductance [4]. In addition, IP3 was extrapolated as $25,23,24,24 \mathrm{dBm}$ for $V_{G S}=-3,-3.5,-4$, and $-4.5 \mathrm{~V}$, respectively. The results suggest that AlGaN/GaN MODFETs can maintain good linearity over a wide range of gate biasing conditions.

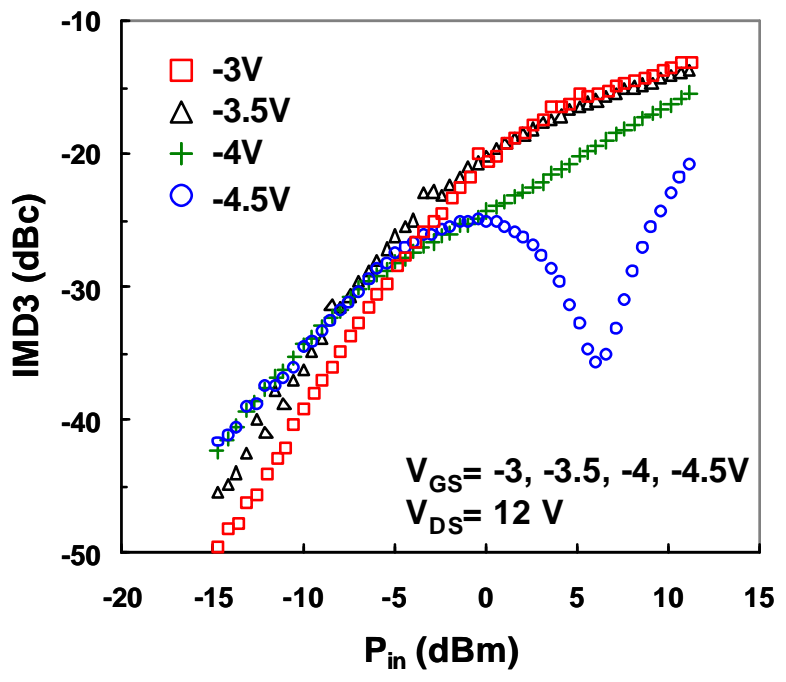

Figure 6: IMD3 with respect to the fundamental output power under different gate bias conditions.

\section{SIMULATION AND DISCUSSION}

The large-signal model of HP ADS was used to simulate the device power characteristics. This model includes dispersion effects, self-heating effects, and includes a gate charge feature for more precise gate capacitance modeling. In addition, this model provides freedom of using different values for DC and AC parameters, such as transconductance and output conductance. The DC parameters were obtained from the device $I-V$ and transconductance characteristics and the parasitic components such as pad capacitance were extracted using device cold S-parameters [5]. Finally, Sparameters under multi-bias points were used to optimize the device parameters simultaneously. Fig. 7 shows the measured and modeled $I-V$ characteristics of AlGaN/GaN MODFETs. As can be seen, very good agreements were obtained. In addition, excellent agreements between measured and modeled S-parameters were achieved in a wide range of bias points. 


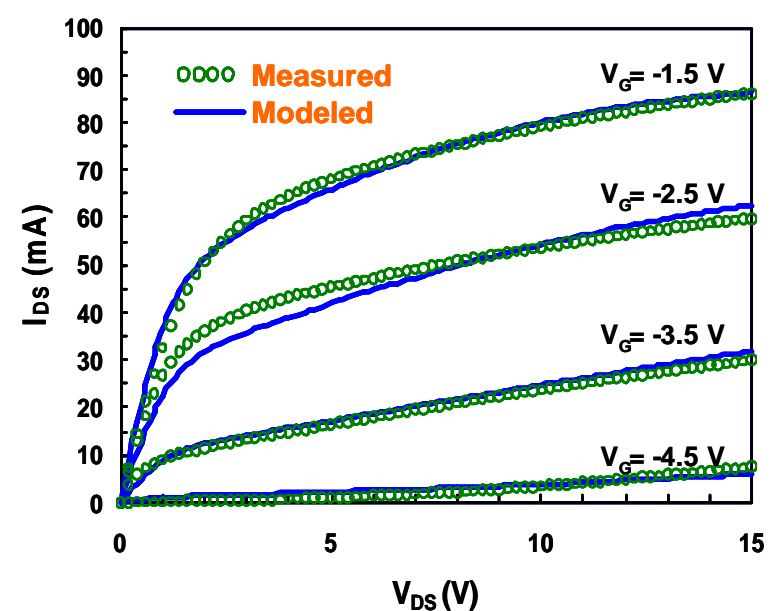

Figure 7: Measured and Modeled I-V characteristics of AlGaN/GaN MODFETs.

Fig. 8 shows the simulation results of $\mathrm{P}_{\text {out }}$, PAE, and $I_{D S}$ with and without harmonic tuners. The simulation was performed using the developed large-signal model and a harmonic balance approach with seven orders of harmonic frequencies. As mentioned above, an improved PAE, a similar $\mathrm{P}_{\text {out }}$, and a reduced drain current level are observed when the load-pull measurement is performed with harmonic tuners. The simulated results show similar trends with the reported experimental results, confirming that the reduced drain current level is a key point in improving PAE.

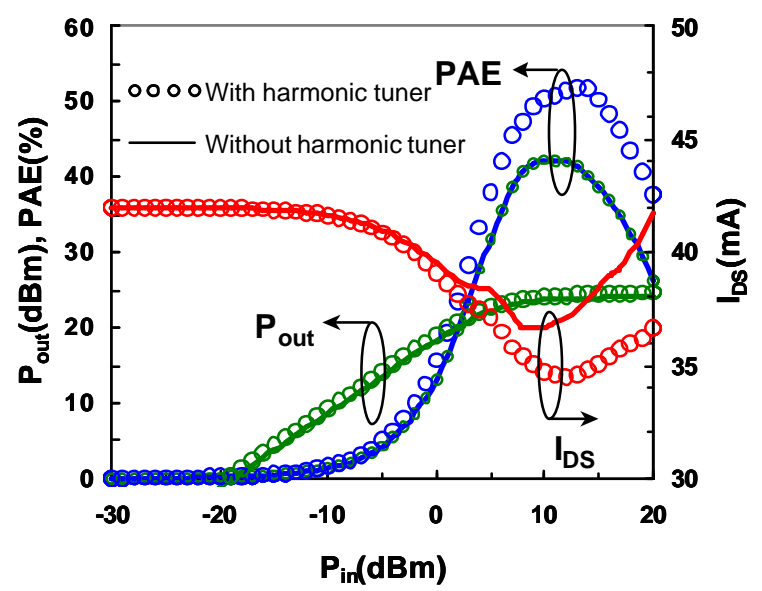

Figure 8: Modeled PAE, $P_{\text {out }}$ and $I_{D S}$ using harmonic balance simulation without and with harmonic terminations. $V_{G S}=-3 \mathrm{~V}, V_{D S}=12 \mathrm{~V}$.

\section{CONCLUSION}

The microwave power characteristics of AlGaN/GaN MODFETs were investigated and the linearity and efficiency of devices under various harmonic termination conditions and bias were analyzed. By use of harmonic tuning, AlGaN/GaN MODFETs can achieve high PAE while keeping a good linearity. In addition, the devices showed best PAE and $\mathrm{P}_{\text {out }}$ at similar output impedances. Measurements of IMD3 and IP3 indicate that the devices maintain good linearity under different gate bias voltages. A large-signal model was used to study the device power characteristics. Results from harmonic balance simulation suggest that the improvement of PAE due to harmonic tuning can mainly be attributed to the change of the effective bias current.

\section{ACKNOWLEDGEMENT}

The authors are grateful to Drs Harry Dietrich and John Zolper for their support and continuous encouragement. Work supported by ONR (contract no.N00014-00-1-0879) and HRL Laboratories.

\section{REFERENCES}

[1] L. Shen, S. Heikman, B. Moran, R. Coffie, N.-Q Zhang, D. Buttari, I. P. Smorchkova, S. Keller, S. P. DenBaars, U.K. Mishra , "AlGaN/AlN/GaN high-power microwave HEMT," IEEE Electron Device Letters, vol. 22, no. 10, pp. 457-459, Oct. 2001.

[2] B. M. Green, V. Tilak, S. Lee, H. Kim, J. A. Smart, K. J. Webb, J. R. Shealy, and L. F. Eastman, "High-Power Broad-Band AlGaN/GaN HEMT MMICs on $\mathrm{SiC}$ Substrates," IEEE Trans. Microwave Theory and Tech., vol. 49, no. 12, pp. 2486-2493, Dec. 2001.

[3] C. Duvanaud, S. Dietsche, G. Pataut, and J. Obregon, "High-efficient class F GaAs FET amplifiers operating with very low bias voltages for use in mobile telephones at $1.75 \mathrm{GHz}$," Microwave and Guided wave lett., vol. 3, no. 8, pp. 268-270, Aug. 1993.

[4] J. A. Higgins, and R. L. Kuvas, "Analysis and improvement of intermodulation distortion in GaAs power FET's," IEEE Trans. Microwave Theory and Tech., vol. 28, no. 1, pp. 9-17, Jan. 1980.

[5] G. Dambrine, A. Cappy, F. Heliodore, and E. Playez, "A New method for determining the FET small-signal equivalent circuit," IEEE Trans. Microwave Theory Tech., vol. 36, No. 7, pp. 1151-1159, July 1988. 